\title{
AN INVERSE PROBLEM FOR EVOLUTION INCLUSIONS
}

\author{
BUI AN TON
}

Received 18 May 2001

An inverse problem, the determination of the shape and a convective coefficient on a part of the boundary from partial measurements of the solution, is studied using 2-person optimal control techniques.

\section{Introduction}

Let $H, \mathscr{H}_{j}, \mathscr{U}_{j} ; j=1, \ldots, N$ be Hilbert spaces and let $\varphi$ be a lower semi-continuous (l.s.c.) function from $H \times \prod_{j=1}^{N} \boldsymbol{u}_{j}$ into $\mathbb{R}^{+}$with $\varphi(\cdot ; u)$ convex on $H$.

Consider the initial-value problem

$$
y^{\prime}+\partial \varphi(y ; u)+f(t, y ; u) \ni 0 \quad \text { on }(0, T), y(0)=y_{0}
$$

With some conditions on $\varphi$ and on $f$, the set $\mathscr{R}(u)$ of all "strong" solutions of (1.1) is nonempty. Let $f_{j}$ be mappings of $L^{2}\left(0, T ; \mathscr{H}_{j}\right) \times \mathcal{U}$ into $\mathbb{R}^{+}$and associate with (1.1) the cost functionals

$$
J_{j}(y ; u)=\int_{0}^{T} f_{j}(y(s) ; u) d s, \quad j=1, \ldots, N
$$

with $D(\varphi(\cdot, u)) \subset \mathscr{H}_{j}$ for all $u \in \mathcal{U}=\prod_{j=1}^{N} \boldsymbol{u}_{j}$.

The existence of an open loop of (1.1), (1.2) with $\varphi$ independent of the control $u$, has been established in Ton [7]. With optimal shape design and with inverse problems in mind, we will consider the case when $\varphi$ depends on the control $u$ as it appears in the top order term of the partial differential operators involved in the problems.

Optimal design of domains has been investigated by Barbu and Friedman [1], Canadas et al. [2], Gunzburger and Kim [3], Pironneau [6], and others. Inverse 
problems have been studied by Canadas et al. [2], Lenhart et al. [4], Lenhart and Wilson [5], and others.

In contrast with all the cited works where a single cost functional is involved, we will consider the $N$-person optimal control approach. It is well known that for $N$-control, open and closed loops are two different notions. In this paper, the existence of an open loop of (1.1), (1.2) is established in Section 3, that is, there exists $\tilde{u} \in U$ such that

$$
J_{j}\left(\tilde{y} ; \pi_{j} \tilde{u}, \tilde{u}_{j}\right) \leq J_{j}\left(y ; \pi_{j} \tilde{u}, v_{j}\right), \quad \forall y \in \mathscr{R}\left(\pi_{j} \tilde{u}, v_{j}\right), \forall v_{j} \in U_{j} ; j=1, \ldots, N,
$$

where $U_{j}$ are given compact convex subsets of the control spaces $\boldsymbol{U}_{j}$ and $\pi_{j}$ is the projection of $u$ onto $\prod_{k \neq j}^{N} u_{k}$.

With a cost functional $f_{j}$ defined by

$$
f_{j}(y)=\|y(\cdot, t)-h(\cdot, t)\|_{L^{2}\left(0, T ; L^{2}(\Omega)\right)}^{2},
$$

where $\Omega$ is a proper subset of the domain and where $h$ is a measurement of the solution $y$ of (1.1) in the subdomain $\Omega$, then (1.1), (1.2) becomes an inverse problem.

Applications to parabolic inequalities are carried out in Section 4 and the notation and the main assumptions of the paper are given in Section 2.

\section{Notation and assumptions}

Let $H, \mathscr{H}_{j}, U_{j} ; j=1, \ldots, N$ be Hilbert spaces. The norm in $H$ is denoted by $\|\cdot\|$ and $(\cdot, \cdot)$ is the inner product in the space. Throughout, $U$ is a given compact convex subset of the control space $u=\prod_{j=1}^{N} u_{j}$.

Assumption 2.1. Let $\varphi$ be a mapping of $H \times \mathcal{U}$ into $\mathbb{R}^{+}$. We assume that

(1) for each $u \in \mathcal{U}, D(\varphi(\cdot ; u))$ is dense in $H$;

(2) $\varphi(y ; u)$ is an l.s.c. function from $H \times \mathcal{U}$ into $\mathbb{R}^{+}$and is convex on $H$ for each given $u \in U$;

(3) there exists a positive constant $c$ such that

$$
c\|y\|^{2} \leq \varphi(y ; u), \quad \forall y \in D(\varphi(y ; u)), \forall u \in \mathcal{U} ;
$$

(4) for each positive constant $C$,

$$
\{y: \varphi(y ; u) \leq C\}
$$

is a compact convex subset of $H$ for each given $u \in \mathcal{U}$;

(5) if $u_{n} \rightarrow u$ in $u$, then

$$
\int_{0}^{T} \varphi(y(s) ; u) d s=\lim _{n} \int_{0}^{T} \varphi\left(y(s) ; u_{n}\right) d s, \quad \forall y \in \bigcap_{u_{n} \in \mathcal{U}} D\left(\varphi\left(\cdot ; u_{n}\right)\right) \cap L^{2}(0, T ; H) .
$$


The subdifferential of $\varphi(y ; u)$ at $y$ is the set

$$
\partial \varphi(y ; u)=\{g: g \in H, \varphi(x ; u)-\varphi(y ; u) \geq(g, x-y), \forall x \in D(\varphi(\cdot ; u))\} .
$$

It is known that $A(y ; u)=\partial \varphi(y ; u)$ is maximal monotone in $H$. The images of $A(y ; u)$ are closed, convex subsets of $H$.

Let $f(y ; u)$ be a mapping of $L^{2}(0, T ; H) \times \boldsymbol{U}$ into $L^{2}(0, T ; H)$ satisfying the following assumption.

Assumption 2.2. We assume that there exists a constant $C$ such that

$$
\|f(y ; u)\|_{H}^{2} \leq C\left\{1+\|u\|_{U_{u}}^{2}+\varphi(y ; u)\right\}
$$

for all $y \in D(\varphi(\cdot ; u))$, all $u \in \mathcal{U}$.

Throughout, the set of solutions of (1.1) is denoted by $\mathscr{R}(u)$.

Assumption 2.3. Let $f_{j}$ be mappings of $L^{2}\left(0, T ; \mathscr{H}_{j}\right) \times \mathscr{U}$ into $\mathbb{R}^{+}$. We assume that

(1) $D(\varphi(\cdot ; u)) \subset \mathscr{H}_{j}$ for all $u \in \mathcal{U}$;

(2) suppose that

$$
\begin{aligned}
& \varphi\left(y^{n} ; u^{n}\right)+\left\|\left(y^{n}\right)^{\prime}\right\|_{L^{2}(0, T ; H)} \leq C, \\
& u^{n} \in U,\left\{y^{n}, u^{n}\right\} \longrightarrow\{y, u\} \quad \text { in } L^{2}(0, T ; H) \times U,
\end{aligned}
$$

then

$$
\int_{0}^{T} f_{j}(y ; u) d t=\lim _{n \rightarrow \infty} \int_{0}^{T} f_{j}\left(y^{n} ; u^{n}\right) d t
$$

\section{Open loop control}

The main result of this section is the following theorem.

Theorem 3.1. Let $\varphi, f$ be as in Assumptions 2.1 and 2.2, and let $f_{j}$ be continuous mappings of $L^{2}\left(0, T ; \mathscr{H}_{j}\right) \times \mathcal{U}$ into $\mathbb{R}^{+}$. Suppose that $y_{0} \in D(\varphi(\cdot ; u))$ for all $u \in U$. Then there exists $\{\tilde{y}, \tilde{u}\} \in\left\{L^{2}(0, T ; H) \cap \mathscr{R}(\tilde{u})\right\} \times U$ such that

$$
J_{j}\left(\tilde{y} ; \pi_{j} \tilde{u}, \tilde{u}_{j}\right) \leq J_{j}\left(y ; \pi_{j} \tilde{u}, v_{j}\right), \quad \forall y \in \mathscr{R}\left(\pi_{j} \tilde{u}, v_{j}\right), \forall v_{j} \in U_{j}, j=1, \ldots, N
$$

Moreover, there exists a positive constant $C$, independent of $u$ such that

$$
\begin{gathered}
\operatorname{ess} \sup \varphi(\tilde{y}(t) ; \tilde{u})+\left\|\tilde{y}^{\prime}\right\|_{L^{2}(0, T ; H)}^{2}+\|A(\tilde{y} ; \tilde{u})\|_{L^{2}(0, T ; H)}^{2} \\
\leq C\left\{1+\sup _{u \in U} \varphi\left(y_{0} ; u\right)\right\},
\end{gathered}
$$

where $A(\tilde{y} ; \tilde{u})$ is an element of the set $\partial \varphi(\tilde{y} ; \tilde{u})$.

First, we will show that the set $\mathscr{R}(u)$ is nonempty. 
Theorem 3.2. Suppose all the hypotheses of Theorem 3.1 are satisfied. Then for each given $u \in U$, there exists a solution $y$ of (1.1) with

$$
\left\|y^{\prime}\right\|_{L^{2}(0, T ; H)}^{2}+\|A(y ; u)\|_{L^{2}(0, T ; H)}^{2}+\operatorname{ess} \sup \varphi(y(t) ; u) \leq C\left\{1+\|u\|_{\mathscr{U}}^{2}\right\} .
$$

The constant $C$ is independent of $u$ and $A(y ; u)$ is an element of $\partial \varphi(y ; u)$.

Proof. For a given $u \in U$, the existence of a solution $y$ of (1.1) with

$$
\left\{y, y^{\prime}, A(y ; u)\right\} \in L^{\infty}(0, T ; H) \times\left(L^{2}(0, T ; H)\right)^{2}
$$

is known (cf. Yamada [8]).

We will now establish the estimate of Theorem 3.2. We have

$$
\left(y^{\prime}, \partial \varphi(y ; u)\right)+\|\partial \varphi(y ; u)\|^{2}+(f(y ; u), \partial \varphi(y ; u))=0 .
$$

With our hypotheses on $f$, we get

$$
\frac{d}{d t} \varphi(y ; u)+\|\partial \varphi(y ; u)\|^{2} \leq C\left\{1+\|u\|_{{ }^{2}}^{2}+\varphi(y(t) ; u)\right\} .
$$

It follows from the Gronwall lemma that

$$
\underset{t \in[0, T]}{\operatorname{ess} \sup } \varphi(y(t) ; u)+\|\partial \varphi(y ; u)\|_{L^{2}(0, T ; H)}^{2} \leq C\left\{1+\|u\|_{u}^{2}\right\} .
$$

The different constants $C$ are all independent of $u$.

With the estimate (2.1), we deduce from (1.1) and from Assumption 2.2 that

$$
\left\|y^{\prime}\right\|_{L^{2}(0, T ; H)}^{2} \leq C\left\{1+\|u\|_{u}^{2}\right\} .
$$

The theorem is thus proved.

Set

$$
\mathscr{B}_{C}=\left\{y:\left\|y^{\prime}\right\|_{L^{2}(0, T ; H)}+\sup _{u \in U} \operatorname{ess} \sup \varphi(y ; u) \leq C\left(1+\sup _{u \in U}\|u\|_{u}\right)\right\} .
$$

Consider the evolution inclusion

$$
y^{\prime}+\partial \varphi(y ; u)+f(x ; u) \ni 0 \quad \text { on }(0, T), y(0)=y_{0}
$$

with $x \in \mathscr{B}_{C}$.

In view of Theorem 3.2, inclusion (3.10) has a unique solution which we will write as $y=R(x ; u)$.

Denote by

$$
J_{j}(x ; y ; u)=\int_{0}^{T} f_{j}(y(s) ; u) d s, \quad j=1, \ldots, N,
$$


the cost functionals associated with (3.10) and where $y=R(x ; u)$ is the unique solution of (3.10).

Let

$$
\Psi(x ; u, v)=\sum_{j=1}^{N} J_{j}\left(x ; y_{j} ; \pi_{j} u, v_{j}\right)
$$

where $y_{j}=R\left(x ; \pi_{j} u, v_{j}\right)$.

Lemma 3.3. Suppose all the hypotheses of Theorem 3.1 are satisfied. Then for each given $\{x, u\} \in \mathscr{B}_{C} \times U$, there exists $v^{*} \in U$ such that

$$
\Psi\left(x ; u, v^{*}\right)=d(x ; u)=\inf \{\Psi(x ; u, v): v \in U\} .
$$

Proof. Let $\left\{v^{n}\right\}$ be a minimizing sequence of (3.13) with

$$
d(x ; u) \leq \Psi\left(x ; u, v^{n}\right) \leq d(x ; u)+n^{-1} .
$$

Since $v^{n} \in U$ and $U$ is a compact subset of $U$, we obtain by taking subsequences that $v^{n_{k}} \rightarrow v^{*}$ in $\mathcal{U}$. Let $y_{j}^{n}=R\left(x ; \pi_{j} u, v_{j}^{n}\right)$, then from the estimates of Theorem 3.2 we obtain, by taking subsequences, that

$$
\begin{aligned}
\left\{y_{j}^{n_{k}},\left(y_{j}^{n_{k}}\right)^{\prime}, A\left(y_{j}^{n_{k}} ; \pi_{j} u, v^{n_{k}}\right)\right\} & \\
& \longrightarrow\left\{y_{j}^{*},\left(y_{j}^{*}\right)^{\prime}, \chi_{j}\right\} \quad \text { in } L^{2}(0, T ; H) \times\left(L^{2}(0, T ; H)\right)_{\text {weak }}^{2} .
\end{aligned}
$$

From the definition of subdifferential, we have

$$
\begin{aligned}
\int_{0}^{T} \varphi\left(z(t) ; \pi_{j} u, v_{j}^{n_{k}}\right) d t-\int_{0}^{T} \varphi\left(y_{j}^{n_{k}}(t) ; \pi_{j} u, v_{j}^{n_{k}}\right) d t \\
\quad \geq \int_{0}^{T}\left(A\left(y_{j}^{n_{k}}(t) ; \pi_{j} u, v_{j}^{n_{k}}\right), z-y_{j}^{n_{k}}\right) d t,
\end{aligned}
$$

for all $z \in L^{2}(0, T ; H)$.

It follows from Assumption 2.1 that

$$
\int_{0}^{T} \varphi\left(z(t) ; \pi_{j} u, v_{j}^{*}\right) d t-\int_{0}^{T} \varphi\left(y_{j}^{*}(t) ; \pi_{j} u, v_{j}^{*}\right) d t \geq \int_{0}^{T}\left(\chi_{j}, z-y_{j}^{*}(t)\right) d t .
$$

Hence

$$
\chi_{j}=A\left(y_{j}^{*} ; \pi_{j} u, v_{j}^{*}\right) .
$$

It is clear that $y_{j}^{*}=R\left(x ; \pi_{j} u, v_{j}^{*}\right)$ and thus,

$$
d(x ; u)=\Psi\left(x ; u, v^{*}\right)=\sum_{j=1}^{N} J_{j}\left(x ; y_{j}, \pi_{j} u, v_{j}^{*}\right),
$$

where $y_{j}=R\left(x ; \pi_{j} u, v_{j}^{*}\right)$.

The lemma is proved. 
Let

$$
X(x ; u)=\left\{v^{*}: \Psi\left(x ; u, v^{*}\right) \leq \Psi(x ; u, v), \forall v \in U\right\} .
$$

Lemma 3.4. Let $g_{j}$ be a continuous mapping of $U_{j}$ into $\mathbb{R}^{+}$and suppose that $g_{j}$ is $1-1$. Then there exists a unique $\hat{v} \in X(x ; u)$ such that

$$
g_{j}\left(\hat{v}_{j}\right)=\inf \left\{g_{j}\left(v_{j}^{*}\right): v^{*} \in X(x, u)\right\} .
$$

Proof. The set $X(x ; u)$ is nonempty and with our hypothesis on $g_{j}$, it is clear that

$$
d_{j}(x ; u)=\inf \left\{g_{j}\left(v_{j}^{*}\right): v^{*} \in X(x ; u)\right\}
$$

exists.

Let $v_{j}^{n}$ be a minimizing sequence of the optimization problem (3.22) with

$$
d_{j}(x ; u) \leq g_{j}\left(v_{j}^{n}\right) \leq d_{j}(x ; u)+n^{-1}, \quad j=1, \ldots, N,
$$

and $v^{n} \in X(x, u)$.

Let $y_{j}^{n}=R\left(x ; \pi_{j} u, v_{j}^{n}\right)$ be the unique solution of (3.10) with controls $\left\{\pi_{j} u, v_{j}^{n}\right\}$ and $f\left(x ; \pi_{j} u, v_{j}^{n}\right)$. Then from the estimates of Theorem 3.2, we obtain, by taking subsequences, that

$$
\left\{y_{j}^{n},\left(y_{j}^{n}\right)^{\prime}, A\left(y_{j}^{n} ; \pi_{j} u, v_{j}^{n}\right)\right\} \longrightarrow\left\{\hat{y}_{j}, \hat{y}_{j}^{\prime}, \chi_{j}\right\} \quad \text { in } L^{2}(0, T ; H) \times\left(L^{2}(0, T ; H)\right)_{\text {weak }}^{2} \text {. }
$$

Since $v^{n} \in U$, we get by taking subsequences that $v^{n} \rightarrow \hat{v}$ in $U$.

A proof, as in that of Lemma 3.3, shows that

$$
\chi_{j}=A\left(\hat{y}_{j} ; \pi_{j} u, \hat{v}_{j}\right), \quad \hat{y}_{j}=R\left(x ; \pi_{j} u, \hat{v}_{j}\right) .
$$

Hence $\hat{v} \in X(x ; u)$. We now have

$$
g_{j}\left(\hat{v}_{j}\right)=d_{j}(x ; u)=\inf \left\{g_{j}\left(v_{j}^{*}\right): v^{*} \in X(x ; u)\right\} .
$$

Since $g_{j}$ is $1-1, \hat{v}$ is unique. The lemma is proved.

Let $\mathscr{L}$ be the nonlinear mapping of $\mathscr{S}_{C} \times U$ into $\mathscr{B}_{C} \times U$, defined by

$$
\mathscr{L}(x, u)=\{\hat{y}, \hat{v}\}
$$

where $\hat{v}$ is the element of $U$ given by Lemma 3.4 and $\hat{y}=R\left(x ; \pi_{j} u, \hat{v}_{j}\right)$ is the unique solution of (3.10) with control $\left\{\pi_{j} u, \hat{v}_{j}\right\}$ and $f\left(x ; \pi_{j} u, \hat{v}_{j}\right)$.

Lemma 3.5. Suppose all the hypotheses of Theorem 3.1 are satisfied. Then $\mathscr{L}$, defined by (3.27), has a fixed point, that is, there exists $\{\tilde{y}, \tilde{u}\} \in \mathscr{B}_{C} \times U$ such that $\mathscr{L}(\tilde{y}, \tilde{u})=\{\tilde{y}, \tilde{u}\}$. 
Proof. (1) We now show that $\mathscr{L}$ has a fixed point by applying Schauder's theorem. Since $\mathscr{B}_{C} \times U$ is a compact convex subset of $L^{2}(0, T ; H) \times U$ and since $\mathscr{L}$ takes $\mathscr{B}_{C} \times U$ into itself, it suffices to show that $\mathscr{L}$ is continuous.

(2) Let $\left\{x^{n}, u^{n}\right\}$ be in $\mathscr{B}_{C} \times U$ and let

$$
y_{j}^{n}=R\left(x^{n} ; \pi_{j} u^{n}, \hat{v}_{j}^{n}\right), \quad \hat{v}^{n} \text { as in Lemma 3.4. }
$$

Since $\left\{x^{n} u^{n}\right\} \in \mathscr{B}_{C} \times U$ and $\mathscr{B}_{C} \times U$ is a compact subset of $L^{2}(0, T ; H) \times \mathcal{U}$, there exists a subsequence such that

$$
\left\{x^{n}, u^{n}, \hat{v}^{n}\right\} \longrightarrow\left\{x^{*}, u^{*}, \hat{v}\right\} \quad \text { in } L^{2}(0, T ; H) \times u \times \vartheta
$$

From the estimates of Theorem 3.2, we get

$$
\left\{y_{j}^{n},\left(y_{j}^{n}\right)^{\prime}, A\left(y_{j}^{n} ; u^{n}\right)\right\} \longrightarrow\left\{y_{j}^{*},\left(y_{j}^{*}\right)^{\prime}, \chi_{j}\right\} \quad \text { in } L^{2}(0, T ; H) \times\left(L^{2}(0, T ; H)\right)_{\text {weak }}^{2}
$$

A proof, as in that of Lemma 3.3, shows that

$$
\chi_{j}=A\left(y_{j}^{*} ; u^{*}\right), \quad y_{j}^{*}=R\left(x^{*} ; \pi_{j} u^{*}, \hat{v}_{j}\right) .
$$

(3) We now show that $u^{*} \in X\left(x^{*}, \hat{v}\right)$. Since

$$
\mathscr{L}\left\{u^{n}, x^{n}\right\}=\left\{v^{n}, y^{n}\right\}
$$

it follows from the definition of $\mathscr{L}$ that

$$
\begin{aligned}
\Psi\left(x^{n} ; u^{n}, v^{n}\right) & \leq \Psi\left(x^{n} ; u^{n}, v\right), \quad \forall v \in U, \\
\sum_{j=1}^{N} J_{j}\left(x^{n} ; y_{j}^{n} ; \pi_{j} u^{n}, v_{j}^{n}\right) & \leq \sum_{j=1}^{N} J_{j}\left(x^{n} ; z_{j}^{n} ; \pi_{j} u^{n}, v_{j}\right), \quad \forall v \in U,
\end{aligned}
$$

where $z_{j}^{n}=R\left(x^{n} ; \pi_{j} u^{n}, v_{j}\right)$ is the unique solution of (3.10) with controls $\left\{\pi_{j} u^{n}, v_{j}\right\}$ and $f\left(x^{n} ; \pi_{j} u^{n}, v_{j}\right)$.

Again from the estimates of Theorem 3.2, we deduce as above that

$$
\left\{z_{j}^{n},\left(z_{j}^{n}\right)^{\prime}, A\left(z_{j}^{n} ; u^{n}\right)\right\} \longrightarrow\left\{z_{j}, z_{j}^{\prime}, A\left(z_{j} ; u^{*}\right)\right\} \quad \text { in } L^{2}(0, T ; H) \times\left(L^{2}(0, T ; H)\right)_{\text {weak }}^{2} \text {. }
$$

It then follows from (3.33) that

$$
\sum_{j=1}^{N} J_{j}\left(x^{*} ; y_{j}^{*} ; \pi_{j} u^{*}, \hat{v}_{j}\right) \leq \sum_{j=1}^{N} J_{j}\left(x^{*} ; z_{j} ; \pi_{j} u^{*} ; v_{j}\right), \quad \forall v \in U,
$$

that is,

$$
\Psi\left(x^{*} ; u^{*}, \hat{v}\right) \leq \Psi\left(x^{*} ; u^{*}, v\right), \quad \forall v \in U
$$


42 An inverse problem for evolution inclusions

Hence

$$
d\left(x^{*}, u^{*}\right)=\Psi\left(x^{*} ; u^{*}, \hat{v}\right)=\inf \left\{\Psi\left(x^{*} ; u^{*}, v\right): v \in U\right\} .
$$

Moreover, we have

$$
\lim _{n} g_{j}\left(v_{j}^{n}\right)=g_{j}\left(\hat{v}_{j}\right), \quad j=1, \ldots, N
$$

By hypothesis, $g_{j}$ is $1-1$ and so $\hat{v}$, the unique element of $X\left(x^{*} ; u^{*}\right)$, with

$$
g_{j}\left(\hat{v}_{j}\right)=\inf \left\{g_{j}\left(v_{j}\right): v \in X\left(x^{*} ; u^{*}\right)\right\},
$$

is in $X\left(x^{*} ; u^{*}\right)$. It follows that $\mathscr{L}\left\{x^{*}, u^{*}\right\}=\left\{y^{*}, \hat{v}\right\}$.

The operator $\mathscr{L}$ is continuous and thus, it has a fixed point by Schauder's theorem. The lemma is thus proved.

Proof of Theorem 3.1. Let $\mathscr{L}$ be as in (3.33). Then it follows from Lemma 3.5 that $\mathscr{L}$ has a fixed point, that is, there exists $\{\tilde{y}, \tilde{u}\}$ with

$$
\mathscr{L}\{\tilde{y}, \tilde{u}\}=\{\tilde{y}, \tilde{u}\} .
$$

Thus,

$$
\tilde{y}^{\prime}+A(\tilde{y} ; \tilde{u})+f(\tilde{y} ; \tilde{u})=0 \quad \text { on }(0, T) ; y(0)=y_{0} .
$$

Moreover,

$$
\sum_{j=1}^{N} J_{j}\left(\tilde{y} ; \pi_{j} \tilde{u}, \tilde{u}_{j}\right) \leq \sum_{j=1}^{N} J_{j}\left(y_{j} ; \pi_{j} \tilde{u}, v_{j}\right), \quad \forall y_{j} \in \mathscr{R}\left(\pi_{j} \tilde{u}, v_{j}\right), \quad \forall v \in U .
$$

Take $v=\left(\pi_{j} \tilde{u}, v_{j}\right)$ and we obtain from (3.42) that

$$
J_{j}\left(\tilde{y} ; \pi_{j} \tilde{u}, \tilde{u}_{j}\right) \leq J_{j}\left(y_{j} ; \pi_{j} \tilde{u}, v_{j}\right), \quad \forall y_{j} \in \mathscr{R}\left(\pi_{j} \tilde{u}, v_{j}\right)
$$

Repeating the process $N$ times we get the theorem.

\section{Applications}

In this section, we give some applications of Theorem 3.1 to parabolic initial boundary value problems. For simplicity, we take $N=2$.

Let $G$ be a bounded open subset of $\mathbb{R}^{2}$ with a smooth boundary and let

$$
\begin{aligned}
Q & =G \times(0,2), \quad \Gamma=G \times\{2\}, \\
Q\left(u_{1}\right) & =\left\{(\xi, \eta): \xi \in G, 0<\eta<u_{1}(\xi)\right\},
\end{aligned}
$$

where $u_{1}$ is a continuous function of $G$ into $[1,2]$. The top of the cylinders $Q\left(u_{1}\right)$, $Q$ are

$$
\Gamma\left(u_{1}\right)=\left\{\left(\xi, u_{1}(\xi)\right): \xi \in G\right\}, \quad \Gamma .
$$


Make the change of variable $\zeta=2 \eta / u_{1}$ and set

$$
y(\xi, \eta)=y\left(\xi, \frac{u_{1} \zeta}{2}\right)=Y(\xi, \zeta)
$$

As done in great details in [4, pages 946-948], we get

$$
\nabla^{2} y=\nabla_{\xi, \zeta} F\left(\xi, \zeta ; u_{1}\right) \nabla_{\xi, \zeta} Y(\xi, \zeta)+u_{1}^{-1} F \nabla Y \cdot \nabla u_{1},
$$

where $F\left(\xi, \zeta ; u_{1}\right)$ is the matrix

$$
\left(\begin{array}{ccc}
1 & 0 & -\zeta\left(\partial_{\xi_{1}} u_{1}\right) u_{1}^{-1} \\
0 & 1 & -\zeta\left(\partial_{\xi_{2}} u_{1}\right) u_{1}^{-1} \\
-\zeta\left(\partial_{\xi_{2}} u_{1}\right) u_{1}^{-1} & -\zeta\left(\partial_{\xi_{1}} u_{1}\right) u_{1}^{-1} & \zeta^{2}\left|\nabla u_{1}\right|^{2} u_{1}^{-2}+4 u_{1}^{-2}
\end{array}\right)
$$

Set

$$
\mu\left(u_{1}\right)=2 u_{1}^{-1} \sqrt{1+\left|\nabla u_{1}\right|^{2}}
$$

4.1. An inverse problem for a nonlinear heat equation. Consider the initial boundary value problem

$$
\begin{aligned}
y^{\prime}-\Delta y & =\tilde{f}(y) & & \text { on } Q\left(u_{1}\right) \times(0, T), \\
y & =0 & & \text { on } \partial Q\left(u_{1}\right) / \Gamma \times(0, T), \\
-\frac{\partial y}{\partial n} & \in u_{2} \beta(y) & & \text { on } \Gamma\left(u_{1}\right) \times(0, T), \\
y(\cdot, 0) & =y_{0} & & \text { on } Q\left(u_{1}\right),
\end{aligned}
$$

where $\beta \in \partial j(r)$ and $j(r)$ is an l.s.c. convex function from $\mathbb{R}^{+}$to $[0, \infty]$.

Let

$$
\begin{aligned}
& J_{1}\left(y ; u_{1}, u_{2}\right)=\int_{0}^{T} \int_{G}\left|y\left(\xi, u_{1}(\xi)\right)\right|^{2} d \xi d t \\
& J_{2}\left(y ; u_{1}, u_{2}\right)=\int_{0}^{T} \int_{\Omega}|y-h(\xi, \eta)|^{2} d \xi d \eta d t
\end{aligned}
$$

be the cost functionals associated with (4.7) and let $h$ be the measurement of the solution $y$ of (4.7) in the sub-region $\Omega$.

We denote

$$
U_{j}=\left\{u_{j}:\left\|u_{j}\right\|_{H^{3}(G)} \leq C, 1 \leq u_{1}(\xi) \leq 2,0 \leq u_{2}(\xi) \leq C\right\}
$$

and let $U_{j}=L^{2}(G)$. It is clear that the $U_{j}$ are compact convex subsets of the space of controls $u_{j}$.

We will take

$$
H=L^{2}(Q), \quad \mathscr{H}_{1}=L^{2}(G), \quad \mathscr{H}_{2}=L^{2}(\Omega), \quad \Omega \subset Q .
$$

The main result of this subsection is the following theorem. 
44 An inverse problem for evolution inclusions

Theorem 4.1. Let $y_{0}$ be in $H_{0}^{1}(Q)$ and let $\tilde{f}$ be a continuous function of $y, u$ with

$$
|\tilde{f}(y ; u)| \leq C\{1+|y|+|u|\} .
$$

Let $h$ be a given function in $L^{2}\left(0, T ; L^{2}(\Omega)\right)$ where $\Omega$ is a proper subset of $Q$ and let $j(r)$ be an l.s.c. convex function on $\mathbb{R}$ with values in $[0,+\infty]$. Then there exists

$$
\begin{aligned}
\left\{\hat{y}, \hat{y}^{\prime}, \hat{u}\right\} \in & L^{2}\left(0, T ; H^{1}\left(Q\left(\hat{u}_{1}\right)\right)\right) \cap L^{\infty}\left(0, T ; L^{2}\left(Q\left(\hat{u}_{1}\right)\right)\right) \\
& \times L^{2}\left(0, T ; L^{2}\left(Q\left(\hat{u}_{1}\right)\right)\right) \times U
\end{aligned}
$$

such that $\hat{y}$ is a solution of the initial boundary value problem (4.7) in $Q\left(\hat{u}_{1}\right) \times$ $(0, T)$; and

$$
\begin{array}{ll}
J_{1}\left(\hat{y} ; \hat{u}_{1}, \hat{u}_{2}\right) \leq J_{1}\left(y ; \hat{u}_{1}, v_{2}\right), & \forall v_{2} \in U_{2}, \\
J_{2}\left(\hat{y} ; \hat{u}_{1}, \hat{u}_{2}\right) \leq J_{2}\left(x ; v_{1}, \hat{u}_{2}\right), \quad \forall v_{1} \in U_{1},
\end{array}
$$

where $x, y$ are the solutions of (4.7) with controls $\left\{v_{1}, \hat{u}_{2}\right\},\left\{\hat{u}_{1}, v_{2}\right\}$ in $Q\left(v_{1}\right) \times(0, T)$ and in $Q\left(\hat{u}_{1}\right) \times(0, T)$, respectively.

Problems of type (4.7) arise in the study of heat transfer between solids and gases under nonlinear boundary conditions.

As carried out in [4], we make the change of variable $\zeta=2 u_{1}^{-1} \eta$ and set $y(\xi, \eta)=Y(\xi, \zeta)$. Then (4.7) is transformed into the following problem:

$$
\begin{gathered}
Y^{\prime}-\nabla\left(F\left(u_{1}\right) \cdot \nabla Y\right)+u_{1}^{-1} F \nabla Y \cdot \nabla u_{1}=\tilde{f}(Y, u) \quad \text { on } Q \times(0, T) \\
Y=0 \quad \text { on } \partial Q / \Gamma \times(0, T) \\
-\frac{\partial Y}{\partial n} \in \mu\left(u_{1}\right) u_{2} \beta(Y) \quad \text { on } \Gamma \times(0, T) \\
Y(\cdot, 0)=y_{0} \quad \text { on } Q
\end{gathered}
$$

with cost functionals

$$
\begin{aligned}
& J_{1}\left(Y ; u_{1}, u_{2}\right)=\int_{0}^{T} \int_{G}|Y(\xi, 2)|^{2} d \xi d t \\
& J_{2}\left(Y ; u_{1}, u_{2}\right)=\int_{0}^{T} \int_{\Omega}\left|Y\left(\xi, \frac{2 \eta}{u_{1}}\right)-h(\xi, \eta ; t)\right|^{2} d \xi d \eta d t,
\end{aligned}
$$

where $\mu$ is as in expression (4.6).

Our aim is to find the controls $u_{1}, u_{2}$ so that the solution $y$ of (4.7), if it is unique, is as close to the measurement $h$ in $\Omega$ as possible.

Let $\varphi$ be the mapping of $H \times U_{1} \times U_{2}$ into $\mathbb{R}^{+}$given by

$$
\varphi\left(Y ; u_{1}, u_{2}\right)=\left\{\begin{array}{l}
\frac{1}{2}\|F(u) \nabla Y\|_{L^{2}(Q)}^{2}+\int_{\Gamma} \mu\left(u_{1}\right) u_{2} j(Y) d \sigma, \quad j(Y) \in L^{1}(\Gamma) \\
+\infty, \quad \text { otherwise, }
\end{array}\right.
$$

where $j(r)$ is an l.s.c. convex function from $\mathbb{R}$ to $[0,+\infty]$ with $j(0)=0$. 
By abuse of notation, we will write $y$ for $Y(\xi, \zeta, t)$ when there is no confusion possible.

Lemma 4.2. Let $\varphi$ be as in (4.17). Then $\varphi$ satisfies Assumption 2.1.

Proof. (1) It is clear that $\varphi(y ; u)$ is an l.s.c. function from $H \times U$ into $\mathbb{R}^{+}$and that $C_{0}^{\infty}(Q) \subset D(\varphi(\cdot, u))$ for all $u \in U$.

(2) It was shown in [4, pages 949-952] that

$$
\int_{Q} F(u)|\nabla y|^{2} d \xi d \zeta \geq c\|y\|_{H^{1}(Q)}^{2}
$$

for all $y$ with $F(u) \nabla y \in H, y=0$ on $\partial Q / \Gamma$.

Since $j(r)$ and $\mu$ are both positive functions, we get

$$
c\|y\|_{H^{1}(Q)}^{2} \leq \varphi(y ; u), \quad \forall y \in D(\varphi) .
$$

(3) By the Sobolev imbedding theorem, the set

$$
\{y: \varphi(y ; u) \leq C\}
$$

is a compact subset of $H=L^{2}(Q)$.

(4) Suppose that $u_{1}^{n} \rightarrow u_{1}$ in $H$ with $u_{1}^{n} \in U_{1}$. Since $u_{1}^{n}$ is in $U_{1}$, it follows from the definition of $U_{1}$ and from the Sobolev imbedding theorem that there exists a subsequence such that $u_{1}^{n} \rightarrow u_{1} \in H^{2}(G)$ and in $C^{1}(\bar{G})$.

With $F(u), \mu(u)$ as above, it is trivial to check that we have

$$
\lim _{n \rightarrow \infty} \int_{0}^{T} \varphi\left(y(s) ; u_{1}^{n}\right) d s=\int_{0}^{T} \lim _{n \rightarrow \infty} \varphi\left(y(s) ; u_{1}^{n}\right) d s .
$$

Lemma 4.3. Let $\varphi$ be as in (4.16). Then $\partial \varphi(y ; u)=-\nabla \cdot(F(u) \nabla y)=A(y ; u)$ with

$$
\begin{gathered}
D(A(y ; u))=\{y: \nabla \cdot(F(u) \nabla y) \in H, y=0 \text { on } \partial Q / \Gamma, \\
\left.-\frac{\partial y}{\partial n} \in \mu\left(u_{1}\right) u_{2} \beta(y) \text { on } \Gamma\right\} .
\end{gathered}
$$

Proof. For $y \in H^{1}(Q)$ with $\nabla \cdot F(u) \nabla y$ in $L^{2}(Q)$, we know that $F(u) \nabla y \cdot n \in$ $H^{-1 / 2,2}(\partial Q)$.

Let $A(y ; u)=-\nabla \cdot F(u) \nabla y$ with

$$
\begin{gathered}
D(A(y ; u))=\{y: y \in H, \nabla \cdot(F(u) \nabla y) \in H, y=0 \text { on } \partial Q / \Gamma, \\
\left.-\frac{\partial y}{\partial n} y \in \mu\left(u_{1}\right) u_{2} y \text { on } \Gamma\right\} .
\end{gathered}
$$

We now show that $A$ is maximal monotone on $H$ and that $A \subset \partial \varphi(y ; u)$. 
(1) It is clear that $A(\cdot ; u)$ is monotone in $H$. For $y \in D(A(\cdot ; u))$ and $x \in$ $D(\varphi(\cdot ; u))$, we have

$$
-(\nabla \cdot F(u) \nabla y, x-y)=(F(u) \nabla y, \nabla x-y)-\left\langle\frac{\partial y}{\partial n}, x-y\right\rangle,
$$

where $\langle\cdot, \cdot\rangle$ is the pairing between $H^{-1 / 2,2}(\Gamma)$ and its dual.

It follows that

$$
-(\nabla \cdot F(u) \nabla y, x-y) \leq \varphi(x ; u)-\varphi(y ; u) .
$$

Hence $A(y ; u) \in \partial \varphi(y ; u)$.

(2) To show that $A(y ; u)$ is maximal monotone, it suffices to show that $I+A(\cdot ; u)$ is onto.

Since $\beta(y) \in \partial j(y)$ is maximal monotone, its resolvent operator $(I+\lambda \beta)^{-1}$ is nonexpansive for all $\lambda>0$.

Consider the elliptic boundary value problem

$$
\begin{array}{rlrl}
-\nabla \cdot\left(F(u) \nabla y_{\lambda}\right) & =f \quad \text { on } Q, \quad y_{\lambda}=0 & & \text { on } \partial Q / \Gamma, \\
\mu\left(u_{1}\right) u_{2} y_{\lambda}+\lambda \frac{\partial}{\partial n} y_{\lambda}=\mu\left(u_{1}\right) u_{2}(I+\lambda \beta)^{-1} x & & \text { on } \Gamma .
\end{array}
$$

For $(f, x) \in L^{2}(Q) \times L^{2}(\Gamma)$, there exists a unique solution $y_{\lambda}$ of (4.17) with $y_{\lambda} \in H^{1}(Q)$. Let $L$ be the mapping of $L^{2}(\Gamma)$ into itself given by

$$
L\left(\sqrt{\mu\left(u_{1}\right) u_{2}} x\right)=\left.\sqrt{\mu\left(u_{1}\right) u_{2}} y_{\lambda}\right|_{\Gamma}
$$

(3) We now show that $L$ is a contraction. Let $L$ be as above, then

$$
\int_{Q} F(u)\left|\nabla\left(y_{\lambda}^{1}-y_{\lambda}^{2}\right)\right|^{2}-\left\langle\frac{\partial}{\partial n}\left(y_{\lambda}^{1}-y_{\lambda}^{2}\right), y_{\lambda}^{1}-y_{\lambda}^{2}\right\rangle=0 .
$$

As shown in [4, pages 949 and 952] we have

$$
c\left\|y_{\lambda}^{1}-y_{\lambda}^{2}\right\|_{H^{1}(Q)}^{2}-\left\langle\frac{\partial}{\partial n}\left(y_{\lambda}^{1}-y_{\lambda}^{2}\right), y_{\lambda}^{1}-y_{\lambda}^{2}\right\rangle \leq 0
$$

Thus,

$$
\begin{aligned}
c \| y_{\lambda}^{1} & -y_{\lambda}^{2}\left\|_{H^{1}(Q)}^{2}+\lambda^{-1}\right\| \sqrt{\mu\left(u_{1}\right) u_{2}}\left(y_{\lambda}^{1}-y_{\lambda}^{2}\right) \|_{L^{2}(\Gamma)}^{2} \\
& \leq \lambda^{-1}\left(\mu\left(u_{1}\right) u_{2}\left\{(I+\lambda \beta)^{-1} x^{1}-(I+\lambda \beta)^{-1} x^{2}\right\}, y_{\lambda}^{1}-y_{\lambda}^{2}\right) \\
& \leq\left\|\sqrt{\mu\left(u_{1}\right) u_{2}} \lambda^{-1}\left(y_{\lambda}^{1}-y_{\lambda}^{2}\right)\right\|_{L^{2}(\Gamma)}\left\|\sqrt{\mu\left(u_{1}\right) u_{2}}\left(x^{1}-x^{2}\right)\right\|_{L^{2}(\Gamma)}
\end{aligned}
$$

We have used the nonexpansive property of $(I+\lambda \beta)^{-1}$ in the above estimate. We know that

$$
a\left\|y_{\lambda}^{1}-y_{\lambda}^{2}\right\|_{L^{2}(\Gamma)}^{2} \leq\left\|y_{\lambda}^{1}-y_{\lambda}^{2}\right\|_{H^{1}(Q)}^{2},
$$

where $a$ is a positive constant. 
Thus,

$$
\begin{aligned}
& \lambda a c\left\|y_{\lambda}^{1}-y_{\lambda}^{2}\right\|_{L^{2}(\Gamma)}^{2}+\left\|\sqrt{\mu\left(u_{1}\right) u_{2}}\left(y_{\lambda}^{1}-y_{\lambda}^{2}\right)\right\|_{L^{2}(\Gamma)} \\
& \quad \leq\left\|\sqrt{\mu\left(u_{1}\right) u_{2}}\left(y_{\lambda}^{1}-y_{\lambda}^{2}\right)\right\|_{L^{2}(\Gamma)}\left\|\sqrt{\mu\left(u_{1}\right) u_{2}}\left(x^{1}-x^{2}\right)\right\|_{L^{2}(\Gamma)} .
\end{aligned}
$$

It follows that

$$
\left\|\sqrt{\mu\left(u_{1}\right) u_{2}}\left(y_{\lambda}^{1}-y_{\lambda}^{2}\right)\right\|_{L^{2}(\Gamma)} \leq \gamma\left\|\sqrt{\mu\left(u_{1}\right) u_{2}}\left(x^{1}-x^{2}\right)\right\|_{L^{2}(\Gamma)}
$$

with

$$
\gamma=\frac{\left\|\mu\left(u_{1}\right) u_{2}\right\|_{L^{\infty}(G)}}{\lambda a c+\left\|\mu\left(u_{1}\right) u_{2}\right\|_{L^{\infty}(G)}}<1 .
$$

Thus, $L$ is a contraction mapping. There exists a unique $y_{\lambda}$ such that

$$
\begin{gathered}
-\nabla \cdot\left(F\left(u_{1}\right) \nabla y_{\lambda}\right)=f \quad \text { on } Q, \\
y_{\lambda}=0 \quad \text { on } \partial Q / \Gamma, \\
\mu\left(u_{1}\right) u_{2} y_{\lambda}+\lambda \frac{\partial y_{\lambda}}{\partial n}=\mu\left(u_{1}\right) u_{2}(I+\lambda \beta)^{-1} y_{\lambda} \quad \text { on } \Gamma .
\end{gathered}
$$

(4) By a standard argument, we get from (4.35) the following estimate:

$$
\left\|y_{\lambda}\right\|_{H^{1}(Q)}^{2} \leq C\|f\|_{L^{2}(Q)} \text {. }
$$

Let $\lambda \rightarrow 0^{+}$, and we get by taking subsequences that $y_{\lambda} \rightarrow y$ in $\left(H^{1}(Q)\right)_{\text {weak }} \cap$ $L^{2}(Q)$. It is clear that $y=0$ on $\partial Q / \Gamma$. On the other hand,

$$
-\frac{\partial y_{\lambda}}{\partial n}=\mu\left(u_{1}\right) u_{2} \lambda^{-1}\left\{I-(I+\lambda \beta)^{-1}\right\} y_{\lambda}=\mu\left(u_{1}\right) u_{2} \beta_{\lambda}\left(y_{\lambda}\right)
$$

where $\beta_{\lambda}$ is the Yosida approximation of $\beta$.

Since

$$
\beta_{\lambda}\left(y_{\lambda}\right) \in \beta\left((I+\lambda \beta)^{-1} y_{\lambda}\right), \quad(I+\lambda \beta)^{-1} y_{\lambda} \longrightarrow y \quad \text { in } L^{2}(\Gamma),
$$

it follows from the maximal monotonicity of $\beta$ that

$$
-\frac{\partial}{\partial n} y \in \mu\left(u_{1}\right) u_{2} \beta(y)
$$

The lemma is proved.

Proof of Theorem 4.1. Consider the optimal control problem

$$
\begin{array}{ll}
Y^{\prime}-\nabla \cdot(F(u) \nabla Y)+g(Y ; u)=0 & \text { on } Q \times(0, T), \\
Y=0 & \text { on }(\partial Q / \Gamma) \times(0, T), \\
-\frac{\partial}{\partial n} Y \in \mu\left(u_{1}\right) u_{2} \beta(Y) & \text { on } \Gamma \times(0, T), \\
Y(\cdot, 0)=y_{0} & \text { on } Q
\end{array}
$$


with

$$
g(Y ; u)=-u_{1}^{-1} F\left(u_{1}\right) \nabla Y \cdot \nabla u_{1}-\tilde{f}(Y, u)
$$

and cost functionals

$$
\begin{aligned}
& J_{1}\left(Y ; u_{1}, u_{2}\right)=\int_{0}^{T} \int_{G}|Y(\xi, 2 ; t)|^{2} d \xi d t \\
& J_{2}\left(Y ; u_{1}, u_{2}\right)=\int_{0}^{T} \int_{\Omega}\left|Y\left(\xi, \frac{2 \eta}{u_{1}}, t\right)-h(\xi, \eta, t)\right|^{2} d \xi d \eta d t
\end{aligned}
$$

It is easy to check that $g$ and $J_{1}, J_{2}$ satisfy Assumptions 2.2 and 2.3, respectively. It follows from Lemmas 4.2 and 4.3 and from Theorem 3.1 that there exists an open loop control $\tilde{u}$ of (4.36) and (4.40), that is, we have

$$
\begin{gathered}
\tilde{Y} \in L^{2}\left(0, T ; H^{1}(Q)\right) \cap L^{\infty}\left(0, T ; L^{2}(Q)\right), \\
\left\{\tilde{Y}^{\prime}, A(\tilde{Y} ; \tilde{u})\right\} \in\left(L^{2}\left(0, T ; L^{2}(Q)\right)\right)^{2},
\end{gathered}
$$

solution of (4.36) with controls $\tilde{u}$. Moreover,

$$
\begin{aligned}
& J_{1}\left(\tilde{Y} ; \tilde{u}_{1}, \tilde{u}_{2}\right) \leq J_{1}\left(y ; \tilde{u}_{1}, v_{2}\right), \\
& J_{2}\left(\tilde{Y} ; \tilde{u}_{1}, \tilde{u}_{2}\right) \leq J_{2}\left(x ; \tilde{u}_{1}, \tilde{u}_{2}\right),
\end{aligned}
$$

for all $y \in \mathscr{R}\left(\tilde{u}_{1}, v_{2}\right)$, for all $v_{2} \in U_{2}$, all $x \in \mathscr{R}\left(u_{1}, \tilde{u}_{2}\right)$, and all $u_{1} \in U_{1}$.

Now set

$$
\hat{y}(\xi, \eta)=\tilde{Y}(\xi, \zeta)=\tilde{Y}\left(\xi, \frac{2 \eta}{\tilde{u}_{1}}\right)
$$

and we get the stated result.

4.2. Parabolic variational inequalities. Consider the initial boundary value problem

$$
\begin{array}{ll}
y^{\prime}-\Delta y=\tilde{f}(y) & \text { on } Q\left(u_{1}\right) \times(0, T), \\
y=0 & \text { on }(\partial Q / \Gamma) \times(0, T), \\
y(\cdot, t) \geq u_{2}(\xi) & \text { on } \Gamma \times(0, T), \\
y(\cdot, 0)=y_{0} & \text { on } Q
\end{array}
$$

with cost functionals

$$
\begin{aligned}
& J_{1}\left(y ; u_{1}, u_{2}\right)=\int_{0}^{T} \int_{G}\left|y\left(\xi, u_{1}(\xi) ; t\right)\right|^{2} d \xi, \\
& J_{2}\left(y ; u_{1}, u_{2}\right)=\int_{0}^{T} \int_{\Omega}|y(\xi, \eta ; t)-h(\xi, \eta)|^{2} d \xi d \eta d t,
\end{aligned}
$$


where $h$ is the partial measurement of the solution $y$ of (4.46) in the subdomain $\Omega \times(0, T), U_{1}$ is as before and

$$
U_{2}=\left\{v:\|v\|_{H^{3}(G)} \leq C, 0 \leq v \text { on } G\right\} .
$$

The main result of this subsection is the following theorem.

THEOREM 4.4. Let $y_{0}$ be an element of $H^{1}(Q)$ with

$$
y_{0}=0 \quad \text { on } \frac{\partial Q}{\Gamma}, \quad y_{0} \geq v \geq 0 \quad \text { on } \Gamma, \forall v \in U_{2}
$$

Let $h \in L^{2}\left(0, T ; L^{2}(\Omega)\right)$ where $\Omega$ is a proper subset of $Q\left(u_{1}\right)$ for all $u_{1} \in U_{1}$ and let $\tilde{f}$ be as in Assumption 2.2. Then there exists

$$
\begin{aligned}
\left\{\hat{y}, \hat{y}^{\prime}, \hat{u}\right\} \in & L^{2}\left(0, T ; H^{1}\left(Q\left(\hat{u}_{1}\right)\right)\right) \cap L^{\infty}\left(0, T ; L^{2}\left(Q\left(\hat{u}_{1}\right)\right)\right) \\
& \times L^{2}\left(0, T ; L^{2}\left(Q\left(\hat{u}_{1}\right)\right)\right) \times U
\end{aligned}
$$

with

$$
\begin{aligned}
& J_{1}\left(\hat{y} ; \hat{u}_{1}, \hat{u}_{2}\right) \leq J_{1}\left(y ; \hat{u}_{1} ; v_{2}\right), \\
& J_{2}\left(\hat{y} ; \hat{u}_{1}, \hat{u}_{2}\right) \leq J_{2}\left(x ; u_{1}, \hat{u}_{2}\right),
\end{aligned}
$$

for all solutions $y$ of (4.46) with controls $\hat{u}_{1}, v_{2}$ all solutions $x$ of (4.42) with controls $u_{1}, \hat{u}_{2}$ and all $\left\{u_{1}, v_{2}\right\} \in U_{1} \times U_{2}$.

As before, we make the change of variables $\zeta=2 \eta / u_{1}$ and as in Section 4.1, we transform (4.42) into a problem in a fixed domain

$$
\begin{gathered}
Y^{\prime}-\nabla \cdot F\left(\left(u_{1}\right) \nabla Y\right)=\tilde{f}(Y, u)+u^{-1} F\left(u_{1}\right) \nabla Y \cdot \nabla u_{1} \quad \text { on } Q \times(0, T), \\
Y=0 \quad \text { on } \partial Q / \Gamma \times(0, T), \\
Y \geq u_{2} \quad \text { a.e. on } \Gamma \times(0, T), \\
Y(\cdot, 0)=y_{0} \quad \text { on } Q .
\end{gathered}
$$

The cost functionals become

$$
\begin{aligned}
& J_{1}\left(Y ; u_{1}, u_{2}\right)=\int_{0}^{T} \int_{G}|Y(\xi, 2 ; t)|^{2} d \xi d t \\
& J_{2}\left(Y ; u_{1}, u_{2}\right)=\int_{0}^{T} \int_{\Omega}\left|Y\left(\xi, \frac{2 \eta}{u_{1}} ; t\right)-h(\xi, \eta ; t)\right|^{2} d \xi d \eta d t .
\end{aligned}
$$

Set

$$
K\left(u_{2}\right)=\left\{y: y \in L^{2}\left(0, T ; L^{2}(Q)\right), y \geq u_{2} \text { a.e. on } \Gamma \times(0, T)\right\} .
$$


Then $K\left(u_{2}\right)$ is a closed convex subset of $L^{2}(0, T ; H)$. Let

$$
\varphi(y ; u)=\frac{1}{2} \int_{0}^{T} \int_{Q} F(u)|\nabla y|^{2} d \xi d \zeta d t+I_{K\left(u_{2}\right)}(y),
$$

where $I_{K\left(u_{2}\right)}$ is the indicator function of the closed convex set $K\left(u_{2}\right)$ of $L^{2}(0, T ; H)$ and

$$
\begin{gathered}
D(\varphi(y ; u))=\left\{y: y \in L^{2}\left(0, T ; H^{1}(Q)\right), y=0 \text { on }(\partial Q / \Gamma) \times(0, T),\right. \\
\left.y \geq u_{2} \text { on } \Gamma \times(0, T)\right\} .
\end{gathered}
$$

Lemma 4.5. Let $\varphi$ be as in (4.53). Then $\varphi$ satisfies Assumption 2.1.

Proof. As in the proof of Lemma 4.2, we have

$$
\varphi(y ; u) \geq c\|y\|_{H^{1}(Q)}^{2}, \quad \forall y \in D(\varphi(\cdot, u)) .
$$

It is clear that

$$
\partial \varphi(y ; u)=\nabla(F(u) \cdot \nabla y)+\partial I_{K\left(u_{2}\right)}(y)
$$

All the other conditions of Assumption 2.1 can be verified without any difficulty.

Lemma 4.6. Suppose all the hypotheses of Theorem 4.4 are satisfied. Then there exists a solution $\tilde{Y}$ of

$$
\begin{aligned}
\tilde{Y}^{\prime}+\partial \varphi(\tilde{Y} ; \tilde{u}) \ni \tilde{f}(\tilde{Y}, \tilde{u}) & +\tilde{u}_{1}^{-1} F\left(\tilde{u}_{1}\right) \nabla \tilde{Y} \cdot \nabla \tilde{u}_{1}, \quad \tilde{Y}(\cdot, 0)=y_{0}, \\
\left\{\tilde{Y}, \tilde{Y}^{\prime}, \partial \varphi(\tilde{Y} ; \tilde{u}), \cdot \tilde{u}\right\} \in & \left(L^{2}\left(0, T ; H^{1}(Q)\right) \cap L^{\infty}\left(0, T ; L^{2}(Q)\right)\right) \\
& \times\left(L^{2}\left(0, T ; L^{2}(Q)\right)\right)^{2} \times U .
\end{aligned}
$$

Moreover,

$$
\begin{aligned}
& J_{1}\left(\tilde{Y} ; \tilde{u}_{1}, \tilde{u}_{2}\right) \leq J_{1}\left(y ; \tilde{u}_{1}, v_{2}\right), \\
& J_{2}\left(\tilde{Y} ; \tilde{u}_{1}, \tilde{u}_{2}\right) \leq J_{2}\left(x ; \tilde{u}_{1}, \tilde{u}_{2}\right),
\end{aligned}
$$

for all solutions $y, x$ of (4.55) with controls $\left\{\tilde{u}_{1}, v_{2}\right\},\left\{u_{1}, \tilde{u}_{2}\right\}$, respectively, and for all $\left\{u_{1}, v_{2}\right\}$ in $U_{1} \times U_{2}$.

Proof. The proof is an immediate consequence of Theorem 3.1 and Lemma 4.5.

Proof of Theorem 4.4. Let $\{\tilde{Y}, \tilde{u}\}$ be as in Lemma 4.6 and set $\hat{y}(\xi, \eta ; t)=$ $\tilde{Y}\left(\xi, 2 \eta / \tilde{u}_{1}\right)$. Then $\hat{y}, \tilde{u}$ is a solution of (4.52) and (4.53). The theorem is proved. 


\section{References}

[1] V. Barbu and A. Friedman, Optimal design of domains with free-boundary problems, SIAM J. Control Optim. 29 (1991), no. 3, 623-637.

[2] G. Canadas, F. Chapel, M. Cuer, and J. Zolésio, Shape interfaces in an inverse problem related to the wave equation, Inverse Problems: An Interdisciplinary Study (Montpellier, 1986), Adv. Electron. Electron Phys., Suppl., vol. 19, Academic Press, London, 1987, pp. 533-551.

[3] M. D. Gunzburger and H. Kim, Existence of an optimal solution of a shape control problem for the stationary Navier-Stokes equations, SIAM J. Control Optim. 36 (1998), no. 3, 895-909.

[4] S. Lenhart, V. Protopopescu, and J. Yong, Identification of boundary shape and reflectivity in a wave equation by optimal control techniques, Differential Integral Equations 13 (2000), no. 7-9, 941-972.

[5] S. Lenhart and D. G. Wilson, Optimal control of a heat transfer problem with convective boundary condition, J. Optim. Theory Appl. 79 (1993), no. 3, 581-597.

[6] O. Pironneau, Optimal Shape Design for Elliptic Systems, Springer Series in Computational Physics, Springer-Verlag, New York, 1984.

[7] B. A. Ton, An open loop equilibrium strategy for quasi-variational inequalities and for constrained non-cooperative games, Numer. Funct. Anal. Optim. 17 (1996), no. 910, 1053-1091.

[8] Y. Yamada, On evolution equations generated by subdifferential operators, J. Fac. Sci. Univ. Tokyo Sect. IA Math. 23 (1976), no. 3, 491-515.

Bui An Ton: Department of Mathematics, University of British Columbia, Vancouver, BC, Canada V6T $1 \mathrm{Z2}$

E-mail address: bui@math.ubc.ca 


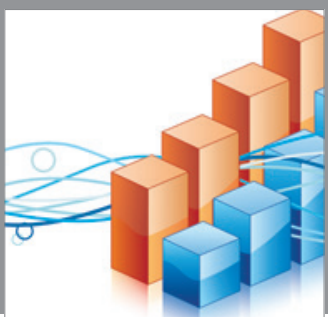

Advances in

Operations Research

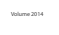

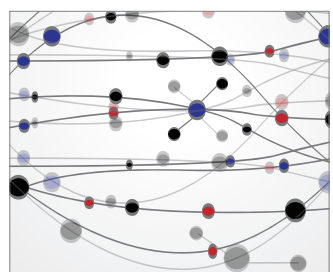

\section{The Scientific} World Journal
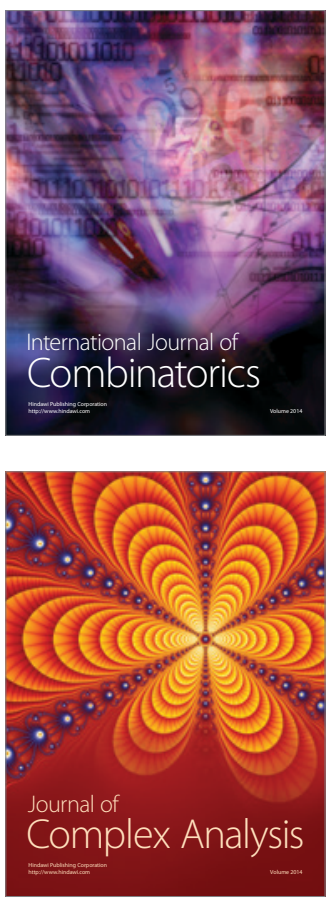

International Journal of

Mathematics and

Mathematical

Sciences
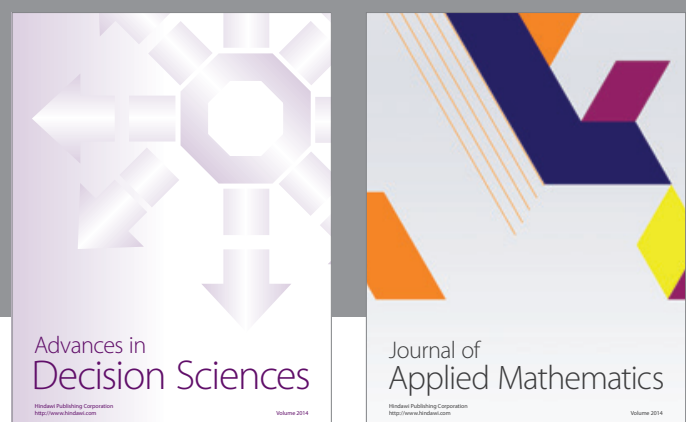

Journal of

Applied Mathematics
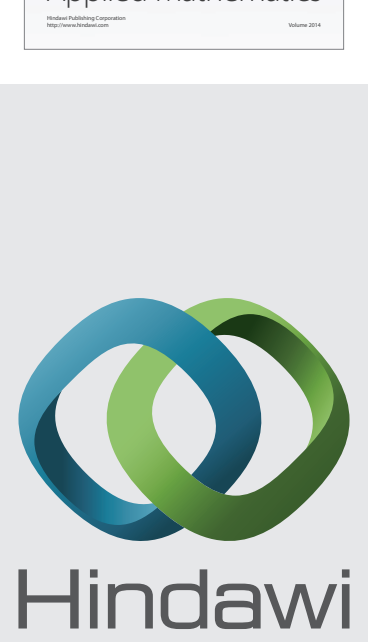

Submit your manuscripts at http://www.hindawi.com
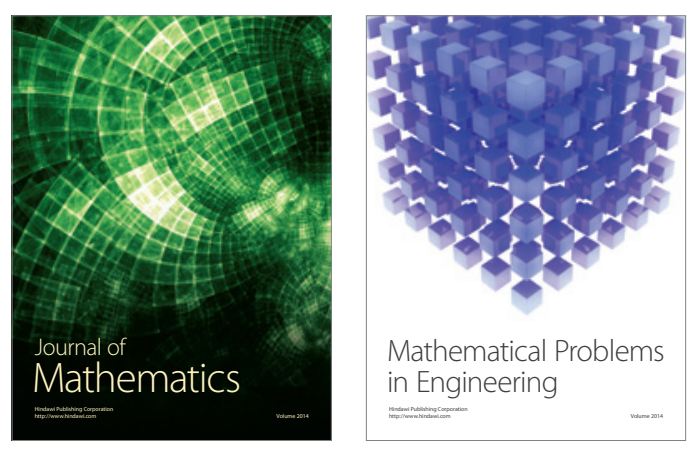

Mathematical Problems in Engineering
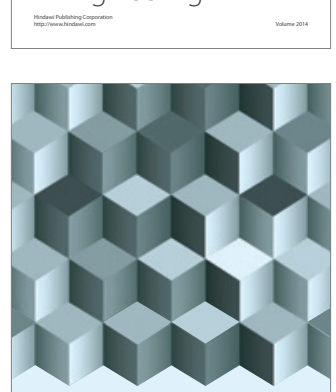

Journal of

Function Spaces
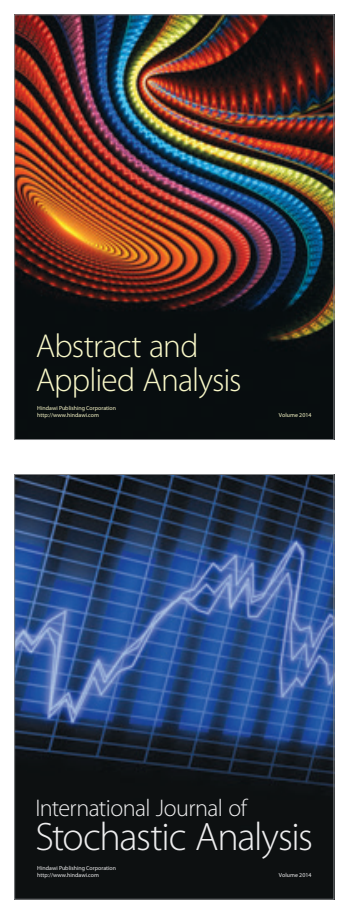

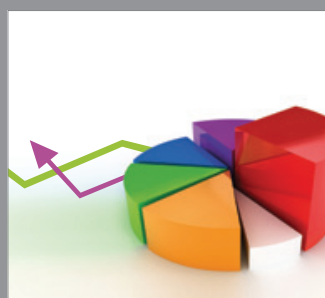

ournal of

Probability and Statistics

Promensencen
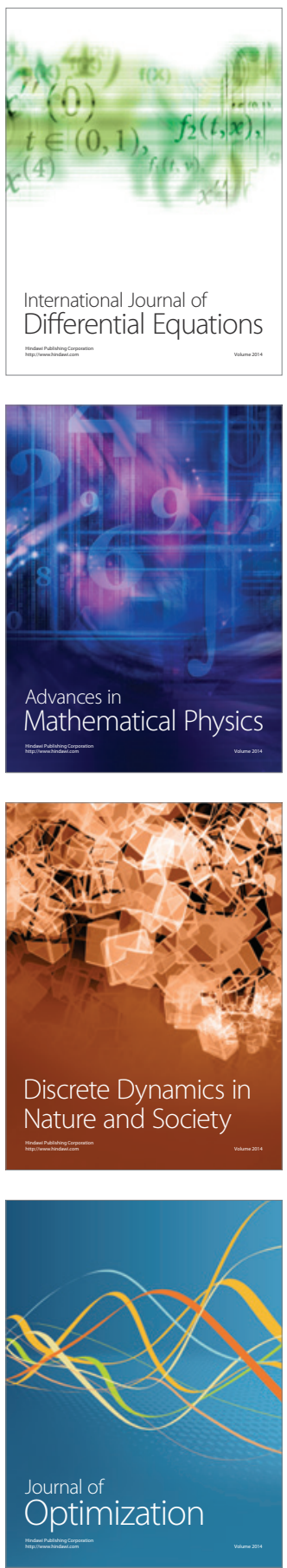